\title{
X-ray Topographic Investigations of Domain Structure in Czochralski Grown $\operatorname{Pr}_{x} \mathrm{La}_{1-x} \mathrm{AlO}_{3}$ Crystals
}

\author{
K. Wieteska ${ }^{a}$, W. Wierzchowski ${ }^{b, *}$, A. MAlinowska ${ }^{b}$, S. TurczyŃski $^{b}$, \\ M. Lefeld-SOSNOWSKA ${ }^{c}$, D.A. PAWLAK ${ }^{b}$, T. EUKASIEWICZ ${ }^{b}$ AND W. GRAefF ${ }^{d}$ \\ ${ }^{a}$ Institute of Atomic Energy POLATOM, 05-400 Otwock-Świerk, Poland \\ ${ }^{b}$ Institute of Electronic Materials Technology, Wólczyńska 133, 01-919 Warsaw, Poland \\ ${ }^{c}$ Institute of Experimental Physics, University of Warsaw, Hoża 69, 00-681 Warsaw, Poland \\ ${ }^{d}$ HASYLAB at DESY, Notkestr. 85, 22-603 Hamburg, Germany
}

\begin{abstract}
In the present paper X-ray diffraction topographic techniques were applied to a number of samples cut from Czochralski grown $\operatorname{Pr}_{x} \mathrm{La}_{1-x} \mathrm{AlO}_{3}$ crystals with different ratio of praseodymium and lanthanum. Conventional and synchrotron X-ray topographic investigations revealed differently developed domain structures dependent on the composition of mixed praseodymium lanthanum aluminium perovskites. Some large mosaic blocks were observed together with the domains. In the best crystals, X-ray topographs revealed striation fringes and individual dislocations inside large domains. Synchrotron topographs allowed us to indicate that the domains correspond to three different crystallographic planes, and to evaluate the lattice misorientation between domains in the range of $20-50$ arc min.
\end{abstract}

PACS numbers: 81.05.-t, 81.10.Dn, 61.72.Ff

\section{Introduction}

The praseodymium lanthanum aluminium perovskite $\mathrm{PrAlO}_{3}$ is a new potential material in visible light laser technology. It is also very interesting in view of the complicated phase transitions, some of them ascribed to the ion lattice coupling. One of the aspects of the interest is connected with the preparation of crystals with self-organized domain structure, with possible application in light guiding [1]. The open problem in the technology of these crystals is the characterisation including examination of the geometrical and crystallographic features of the observed domain structure, and the presence of other crystallographic defects, as dislocations and inclusions.

Domains have a structural character. At room temperature $\mathrm{PrAlO}_{3}$ shows a simple paramagnetic behaviour. Only at low temperatures some anomalous magnetic properties are observed, where reorientation of structural domains with the magnetic field can be indicated [2-4]. Similar kind of domain structure can be observed also in parent compounds, as e.g., $\mathrm{LaAlO}_{3}$ and $\mathrm{NdAlO}_{3}$. Some control over the domain structure can be possible, i.e., a way to detwin [5] the $\mathrm{LaAlO}_{3}$ crystal has been described previously, as well as, the possibility of the growth of thin single crystalline fibres, which did not exhibit twin structure, has been also presented for $\mathrm{LaAlO}_{3}$ crystal [6]. However, the way of control of the domain width has not been proposed yet.

$\mathrm{PrAlO}_{3}$ similarly as $\mathrm{LaAlO}_{3}$ is a rhombohedrally distorted perovskite (space group $R \overline{3} c$, tilt system $\boldsymbol{a a a}$ ) [7-11]. Phase transitions of $\mathrm{PrAlO}_{3}$ can be described as $C 2 / m \stackrel{150 \mathrm{~K}}{\longrightarrow} \operatorname{Imma} \stackrel{205 \mathrm{~K}}{\longrightarrow} R \overline{3} c \stackrel{1650 \mathrm{~K}}{\longrightarrow} \operatorname{Pm} \overline{3} m[1]$.

\footnotetext{
* corresponding author; e-mail: wierzc_w@itme.edu.pl
}

In the present paper we applied X-ray diffraction topographic techniques exploring both synchrotron and conventional X-ray sources to a number of samples cut from Czochralski grown $\operatorname{Pr}_{x} \mathrm{La}_{1-x} \mathrm{AlO}_{3}$ crystals with different values of $x$, as well as, from the $\mathrm{LaAlO}_{3}$ crystal doped with 5 at.\% of praseodymium.

The majority of investigated samples contained extended domain structure, expected to be a result of twinning with $\{100\}$ invariant twin planes. The domains are visible using the optical microscopy equipped with polarizers, or with Nomarski phase contrast, but some fine details of the domains were previously studied with the atomic force microscopy (AFM) [1]. The width of particular domains was in the range from tens to hundreds of micrometers.

\section{Experimental}

The samples were cut from $\operatorname{Pr}_{x} \mathrm{La}_{1-x} \mathrm{AlO}_{3}$ (where $0 \leq$ $x \leq 1$ ) crystals with various chemical composition including both the cases when praseodymium replaced part of lanthanum atoms or it was introduced as a dopant. The crystals were grown by the Czochralski method with a preferred orientation along $\langle 112\rangle$ axis. The growth conditions are described elsewhere [12]. Some of the samples were oriented along $\langle 111\rangle$ growth axis. The mechanically and chemically polished samples were studied with a number of X-ray topographic methods exploring both synchrotron and conventional sources.

Synchrotron X-ray topographic experiments were performed using the white beam, mainly in a back reflection geometry; only to some selected samples the transmission geometry was applied. The conventional X-ray Lang topographs were taken in the back reflection geometry for selected samples. 


\section{Results and discussion}

Synchrotron white beam topographs usually reproduced the domain structure as a series of mutually displaced stripes corresponding to different orientation of the crystal inside the domains, which probably are of twin type. Different appearance of the domain structure has been observed in samples differing in the chemical composition.

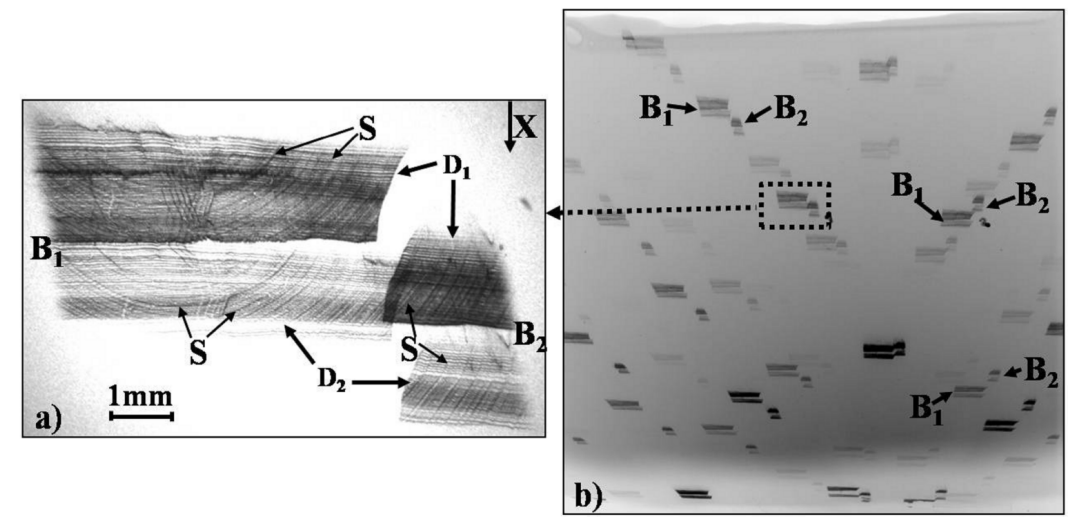

Fig. 2. (a) The white beam back-reflection synchrotron topograph of $\operatorname{Pr}_{0.4} \mathrm{La}_{0.6} \mathrm{AlO}_{3}$ sample reproduced in two systems $\left(\mathrm{D}_{1}\right.$ and $\left.\mathrm{D}_{2}\right)$ of narrow domains. (b) The synchrotron diffraction pattern of the same sample with a larger number of Laue spots. The right part of the topogram in Fig. 2a corresponds to a slightly misoriented grain, located differently with respect to the other part of the Laue spots. $\mathrm{B}_{1}, \mathrm{~B}_{2}-$ misoriented grains, $\mathrm{D}_{1}, \mathrm{D}_{2}-$ domain systems, $\mathrm{S}-$ striations, $\boldsymbol{X}$ - projection of the synchrotron radiation beam direction on the film.

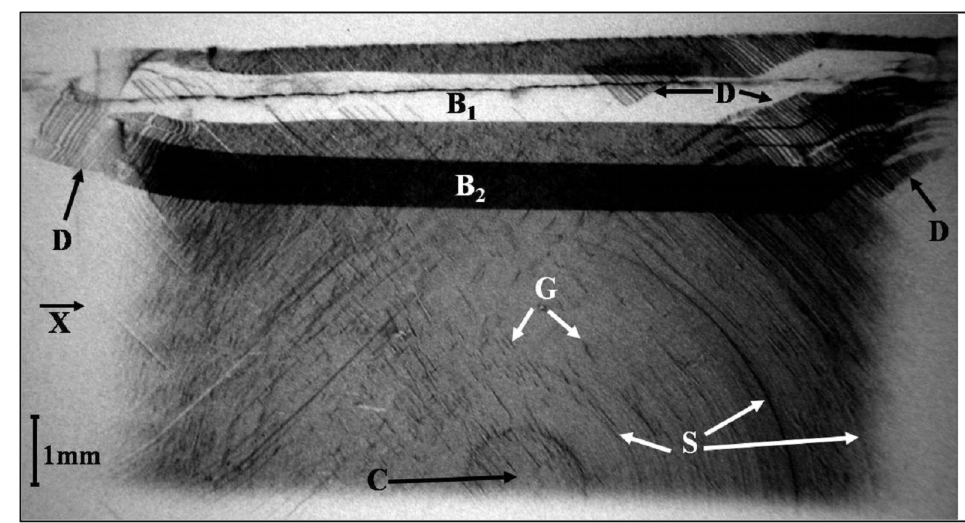

Fig. 4. The representative white beam back-reflection projection topograph of the sample cut from $\mathrm{LaAlO}_{3}$ crystal doped with 5 at. $\%$ Pr. B B $_{2}$ - diffraction contrasts of misoriented block; $\boldsymbol{S}$ - striations fringes; $\boldsymbol{C}$ - crystal core; $\boldsymbol{D}$ - domain systems; $\boldsymbol{G}$ - dislocations, $\boldsymbol{X}$ - projection of the synchrotron radiation beam direction on the film.

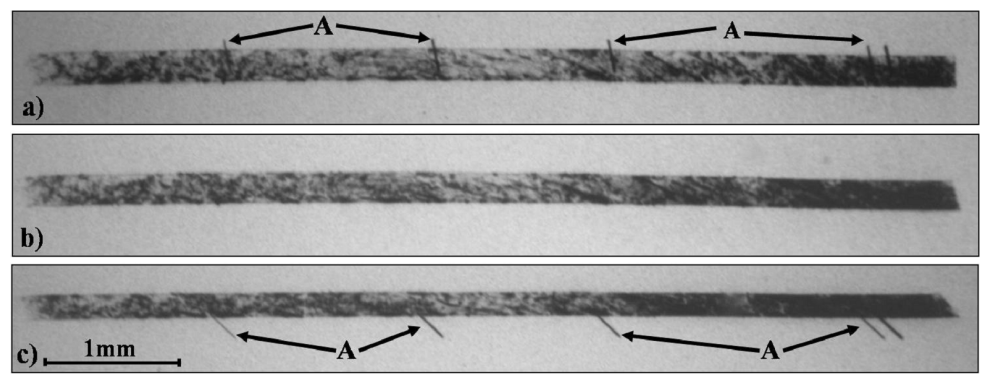

Fig. 5. A set of three transmission section topographs for the same crystal as in the case of Fig. 4, revealing the misorientation of domain regions (a series of displaced stripes, corresponding to different orientation of the crystal inside the domains, marked by $\boldsymbol{A}$ ).

The white beam projection topographs of five representative samples, with different density and extension of the revealed domains, are shown in Figs. 1-6. The topographs of the sample cut from $\mathrm{Pr}_{0.75} \mathrm{La}_{0.25} \mathrm{AlO}_{3}$ crys- 


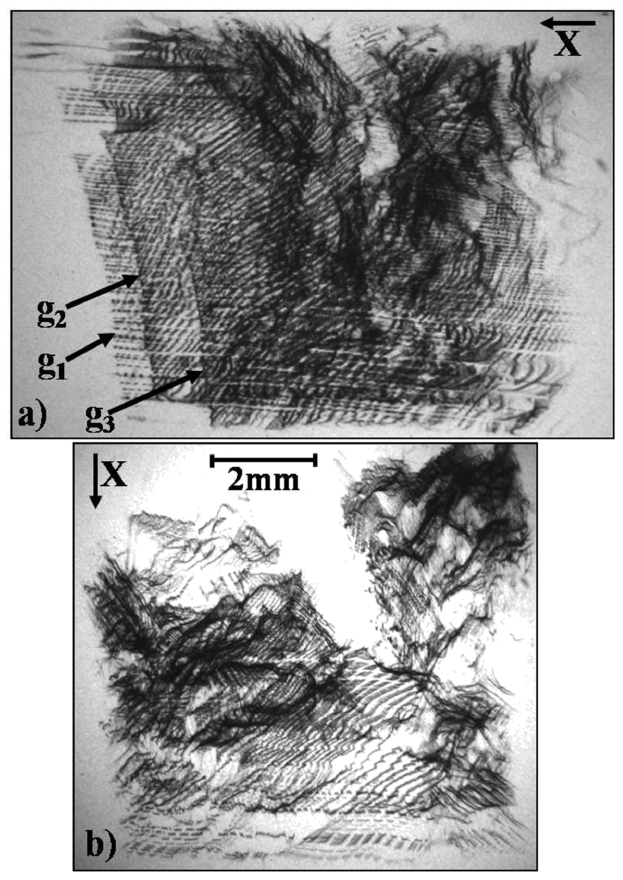

Fig. 1. The representative white beam back-reflection projection topographs (differing through $90^{\circ}$ ) of the sample, cut from $\operatorname{Pr}_{0.75} \mathrm{La}_{0.25} \mathrm{AlO}_{3}$ crystal, with extended domain structure: $g_{1}, g_{2}, g_{3}$ denote the representative boundaries of the same block reproduced by variously oriented domains. $\boldsymbol{X}$ - projection of synchrotron radiation beam direction on the film.

tal, containing one of the most dense and well developed domain structure, are shown in Fig. 1. It may be seen that domains are arranged in three different directions. Apart from the domains we may observe a certain structure of large blocks. As it is seen in the left part in Fig. 1, in some cases, the boundaries of the single block seem to be reproduced at different places by two or more systems of domains as it is representatively marked by $g_{1}, g_{2}, g_{3}$ in Fig. 1a. Some elements of contrast, particularly the bending of some stripes, correspond to the optical images of similar crystals discussed in Ref. [1]. The topograph taken for the second azimuth revealed also three directions of domain contrasts, but the positions of separate blocks are significantly different.

A significantly simpler situation, showing blocks and domain structure, explaining the elements of contrast in Fig. 1, can be seen in Fig. 2a, which presents the back-reflection topograph of $\operatorname{Pr}_{0.4} \mathrm{La}_{0.6} \mathrm{O}_{3}$ sample. The topograph in Fig. 2b shows the whole diffraction pattern with a great number of Laue spots. Each spot consists of two displaced parts, which correspond to the slightly misoriented grains. The images of the right grain are located differently in the each spot. The images of both grains (marked as $\mathrm{B}_{1}$ and $\mathrm{B}_{2}$ in Fig. 2a) consist of two parts (marked as $\mathrm{D}_{1}$ and $\mathrm{D}_{2}$ ), in which the diffraction contrasts - corresponding to sets of differently oriented domains and to segregation fringes — are seen (Fig. 2).

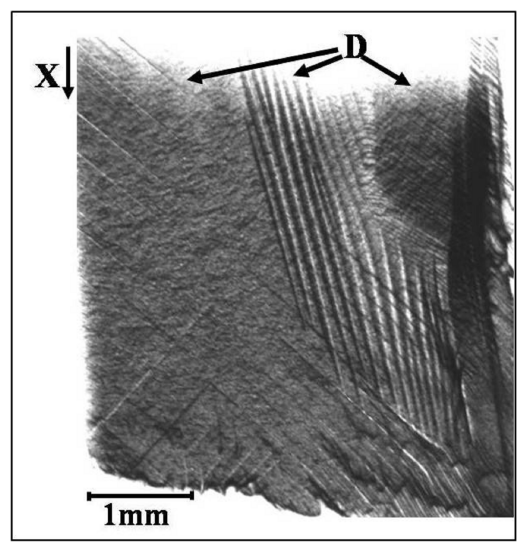

Fig. 3. The representative white beam back-reflection projection topograph of the sample, cut from $\mathrm{LaAlO}_{3}$ crystal, with extended domain structure. D - representative domains systems, $\boldsymbol{X}$ - projection of the synchrotron radiation beam direction on the film.

In the case of undoped $\mathrm{LaAlO}_{3}$ crystal, domain systems located also in three different directions were observed (Fig. 3). However, the domain systems are not dominating the picture, and large areas of more perfect crystal can be indicated. These regions may contain a significant concentration of dislocations or precipitates. Some of visible domain contrasts seem to come directly from mutual misorientation of neighbouring domains, while the others more probably come from domain boundaries. The topograph of $\mathrm{LaAlO}_{3}$ crystal doped with 5 at. $\%$ Pr contains the large regions of considerably good perfection (Fig. 4). The topograph reveals particular striation fringes and a core in the central part of the crystal. Some short linear contrasts may be attributed to individual dislocations of a relatively small density. The longer linear contrasts should, however, be interpreted as domains. Apart from these defects we observed some large misoriented blocks with straight boundaries.

Figure 5 reports the representative synchrotron transmission section topographs of the same sample as in Fig. 4. One can observe five dash formed contrasts in Figs. 5a and 5c appearing on the upper and lower side of the main section image respectively (marked by $\boldsymbol{A}$ ). These dashed contrasts are not visible in Fig. 5b, which most probably corresponds to the reflection coming from the crystallographic plane common for the domains and the matrix crystal.

As it may be seen in Fig. 6, differently than in the synchrotron topographs, the conventional Lang topograph does not reproduce misoriented material of the domains, however, the structure of the whole sample and its block structure are well reproduced.

The visibility of different domains in the synchrotron topographs allowed a rough evaluation of the lattice misorientation. As the mutual displacement revealed in the topographs did not exceed the values of $1-2 \mathrm{~mm}$, the corresponding misorientation reached 20-50 arc minutes. 


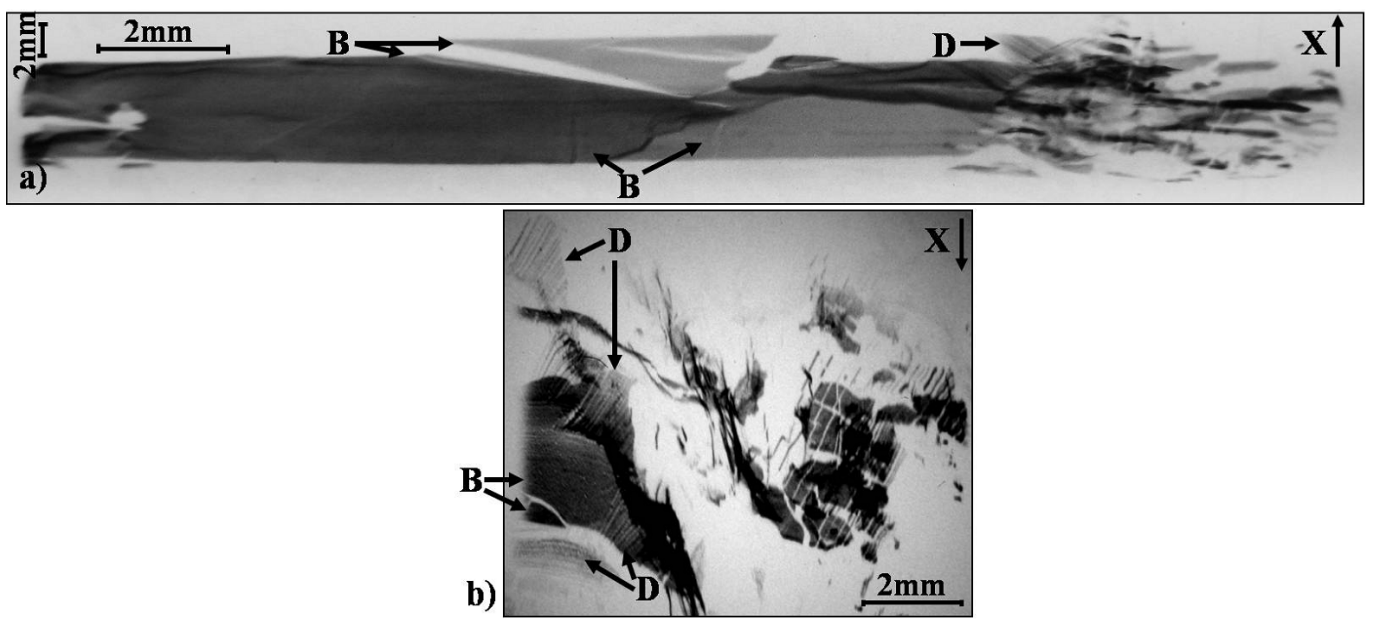

Fig. 6. (a) The Lang back-reflection topograph ((222) reflection for $\mathrm{MoK}_{\alpha 1}$ radiation) of the sample $\mathrm{PrAlO}_{3}$ exhibiting some mosaic blocks and the domains. (b) The white beam synchrotron back-reflection projection topograph of the right part of the sample with extended blocks and domain structures. $\boldsymbol{B}$ - crystal blocks; $\boldsymbol{D}-$ domain systems, $\boldsymbol{X}-$ projection of the incident beam direction on the film.

The density of domains was different in samples cut from various crystals with different composition, but generally it was most numerous in the case of crystals with the highest concentration of praseodymium. In this case, synchrotron topographs usually revealed sets of domains corresponding to more than one system of twinning. It is also probable that in the case of the most dense domain structure, the stripes revealed by topographs correspond rather to some sets of domains than to single domains. The samples with low concentration of praseodymium contained large, more perfect regions without the domains, where it was possible to reveal individual dislocations and segregation fringes.

\section{Conclusions}

Conventional and synchrotron X-ray topographic investigations revealed differently developed domain structures, most probably of twin type, in $\operatorname{Pr}_{x} \mathrm{La}_{1-x} \mathrm{AlO}_{3}$ crystals grown by the Czochralski method, dependent on the La to Pr ions ratio. Apart from the domains some large mosaic blocks were indicated. Striation fringes and individual dislocations were observed in the more perfect crystal regions.

In synchrotron methods, the domains were revealed in topographs at significantly displaced positions. These topographs indicated that domains correspond to three different crystallographic planes and allowed for approximate evaluation of the lattice misorientation, which was in the range of 20-50 arc min.

The density of domains was different in the samples cut from various crystals with different composition, but generally it was the highest in the case of crystals with the highest concentration of praseodymium, where the width of domains revealed by synchrotron topographic methods was in the range of single micrometers. Contrary the size of domains was in the range of single millimetres in case of $\mathrm{LaAlO}_{3}$ crystal doped with 5 at.\% Pr.

The applied X-ray diffraction topographic methods seem to be adequate for further investigations.

\section{Acknowledgments}

Synchrotron investigations were supported by the HASYLAB project II-20060165 EC. The authors thank the Ministry of Science and Higher Education of Poland for support of this work.

\section{References}

[1] D.A. Pawlak, T. Łukasiewicz, M. Carpenter, M. Malinowski, R. Diduszko, J. Kisielewski, J. Cryst. Growth 282, 260 (2005).

[2] A. Riseberg, E. Cohen, W.A. Nordland, L.G. van Uitert, Phys. Lett. A 30, 4 (1969).

[3] M. Wencka, S. Vrtnik, M. Jagodič, Z. Jagličić, S. Turczynski, D.A. Pawlak, J. Dolinšek, Phys. Rev. B $\mathbf{8 0}$, 224410 (2009).

[4] B. Andrzejewski, D.A. Pawlak, T. Klimczuk, S. Turczyński, submitted to Chem. Mat.

[5] H. Fay, C.D. Brandle, J. Appl. Phys. 38, 3405 (1967).

[6] M.R.B. Andreeta, E.R.M. Andreeta, A.C. Hernandes, J. Crystal Growth 275, e757 (2005).

[7] A.M. Glazer, Acta Cryst. B 28, 3384 (1972).

[8] A.M. Glazer, Acta Cryst. A 31, 765 (1975).

[9] H.D. Megaw, C.N.W. Darlington, Acta Cryst. A 31, 165 (1975).

[10] C.J. Howard, B.J. Kennedy, B.C. Chakoumakos, J. Phys., Condens. Matter 12, 349 (2000).

[11] S.M. Moussa, B.J. Kennedy, B.A. Hunter, C.J. Howard, T. Voigt, J. Phys., Condens. Matter 13, L2003 (2001).

[12] S. Turczynski, D.A. Pawlak, R. Diduszko, J. Mucha, M. Pękała, submitted to Cryst. Growth \& Design.. 